\title{
Características de las consultas de los recién nacidos en un servicio de emergencia pediátrica
}

Newborn consultations in a pediatric emergency department

Características das consultas de recém-nascidos em um serviço de emergência pediátrica

Mariana Más ${ }^{1}$, Ana Casuriaga², Gustavo Giachetto ${ }^{3}$, Carina Giacoia ${ }^{4}$, Lucía Centena ${ }^{5}$, Magdalena López ${ }^{4}$, Yelenna Ramírez ${ }^{6}$

\section{Resumen}

Introducción: las consultas de recién nacidos (RN) a servicios de urgencia (SU) son cada vez más frecuentes. Sin embargo, no siempre corresponden a problemas con riesgo significativo.

Objetivo: describir las características de las consultas de los RN en el SU de un prestador integral de salud privada entre el $1 / 1 / 2018$ y el 31/12/2018.

Metodología: estudio descriptivo, mediante revisión de historias clínicas y entrevista telefónica a las madres. Se analizaron variables del RN, de la consulta y maternas.

Resultados: se registraron 237 consultas, correspondientes a 209 RN, sexo masculino 53\%, media de edad 16 días. Los diagnósticos al egreso se agruparon en tres categorías: 1) consultas vinculadas a aspectos de puericultura 29\%,2) procesos fisiológicos $22 \%$ y 3 ) patologías agudas 49\%. La tasa de hospitalización fue $27 \%$. Ingresaron 64 RN: 2 del grupo 1 (escaso incremento ponderal); 6 del grupo 2 (ictericia); 56 del grupo 3. La media de la estadía hospitalaria fue 3 días (rango 1-14). No se registraron fallecimientos.

Conclusiones: predominaron las consultas de RN sin patología aguda, potencialmente evitables. A pesar de ello, debido a la elevada vulnerabilidad de este grupo etario, el pediatra de urgencias debe realizar una evaluación minuciosa en estos niños. Es probable que, facilitando el acceso al primer nivel de atención y completando la información que se brinda a los padres, pueda disminuir la frecuencia de estas consultas. Conocer los problemas de salud de los RN y las principales preocupaciones de los padres constituye un importante insumo para implementar estrategias de mejora y optimizar la utilización de los recursos.

\footnotetext{
1. Jefe de Servicio de Emergencia Pediátrica, Departamento de Atención Integral del niño, niña y adolescente, Asociación Española. Profesora Adjunta Departamento de Emergencia Pediátrica, Facultad de Medicina, UDELAR.

2. Adjunta al Departamento de Atención Integral del niño, niña y adolescente, Asociación Española. Asistente Clínica Pediátrica C, Facultad de Medicina, UDELAR.

3. Director del Departamento de Atención Integral del niño, niña y adolescente, Asociación Española. Profesor Clínica Pediátrica C, Facultad de Medicina, UDELAR.

4. Pediatra, Departamento de Atención Integral del niño, niña y adolescente, Asociación Española.

5. Residente de pediatría, Departamento de Atención Integral del niño, niña y adolescente, Asociación Española.

6. Adjunta al Departamento de Atención Integral del niño, niña y adolescente, Asociación Española.

Aprobado por Comité de Ética de la Asociación Española.

Los autores declaran no tener conflictos de interés. Artículo original e inédito.

Correspondencia: Dra. Ana Casuriaga. Correo electrónico: anitacasuriaga7@gmail.com

Asociación Española.

Recibido: 5/5/2021

Aprobado: $26 / 8 / 2021$

Attribution-NonCommercial 4.0 International (CC BY-NC 4.0)
} 
Palabras clave: Recién nacido

Servicios médicos de urgencia

Pediatría

Key words: Newborn

Emergency medical services

Pediatrics

\section{Introducción}

A nivel mundial, las consultas de los recién nacidos (RN) en los servicios de urgencia (SU) presentan una tendencia creciente en los últimos años. Diversos factores sociales, económicos y culturales se han vinculado con esta tendencia. El alta precoz luego del nacimiento que puede impactar negativamente en la adaptación a los cuidados del RN, el descenso en el número de hijos, la paridad tardía, y las dificultades en el acceso al primer nivel de atención, son algunos de los más implica$\operatorname{dos}^{(1-10)}$

Por otra parte, en diversas comunicaciones se señala que el diagnóstico más frecuente de los RN que consultan en los SU es "recién nacido sin patología". En estos casos, frecuentemente las consultas suelen estar motivadas por inquietudes acerca de los cuidados del RN y condiciones fisiológicas ${ }^{(1,3,8,11)}$. Se trata de un grupo de consultas potencialmente evitables si se conocen las causas que las motivan y se disponen de estrategias para su prevención.

A pesar de ello es necesario considerar que, según algunas series, una proporción importante de los cuidadores consultan con sus RN en los SU en forma justificada por patologías que es necesario reconocer y tratar en forma oportuna ${ }^{(1,11)}$.

El aumento de las consultas neonatales en los SU determina un aumento de la demanda asistencial y, en casos injustificados, contribuye a su saturación con la consiguiente sobrecarga de trabajo e impacto en la calidad asistencial. A la vez representan un desafío para los equipos de profesionales, ya que deben identificar y manejar en forma oportuna las situaciones de riesgo significativo.

En el prestador integral de salud donde se realizó esta investigación (Asociación Española) se dispone de un SU específico para la atención de niños y adolescentes, centralizado que asiste aproximadamente 30.000 consultas anuales. Se utiliza un sistema de triage que prioriza la atención según nivel de urgencia y contribuye a que cada niño sea evaluado en un tiempo y nivel adecuado. Debido a la mayor vulnerabilidad y escasa expresividad clínica de los RN, su evaluación debe ser realizada en forma rápida, completa y minuciosa por personal debidamente entrenado. Es por ello que este grupo etario se clasifica siempre en niveles elevados de prioridad. Esto resulta fundamental además para evitar su exposición a otros niños o adultos con enfermedades agudas e infectocontagiosas en las salas de espera ${ }^{(1,12)}$. En este centro asistencial se registraron 1.579 nacimientos en 2018. Se destaca que a todas las embarazadas se les ofrece instancias de capacitación acerca del trabajo de parto, puerperio, lactancia y puericultura, y al momento del egreso de la unidad neonatal se brindan recomendaciones escritas sobre cuidados del RN, se coordinan los controles en salud del primer mes de vida, y en caso de identificar riesgos, se planifican visitas domiciliarias.

Desde 2016, con el objetivo de contribuir a mejorar la gestión clínica en el SU de este prestador de salud, se han medido varios indicadores de calidad de la asistencia. En este marco, resulta importante conocer las características de las consultas neonatales en este servicio como insumo para el plan de mejora continua.

\section{Objetivos}

\section{Objetivo general}

Describir las características de las consultas de los RN en el SU de un prestador integral de salud privada, entre el $1 / 1 / 2018$ y el $31 / 12 / 2018$.

\section{Objetivos específicos}

Describir las características de los RN que consultaron en el SU en el período de estudio.

Describir algunas características de las madres de los RN que consultaron en el SU en el período de estudio.

\section{Material y métodos}

Se realizó un estudio descriptivo, retrospectivo, en el que se analizaron todas las consultas de los RN (edad $<28$ días) en el SU del prestador de salud integral privado entre el 1 de enero y el 31 de diciembre de 2018.

Se analizaron variables del recién nacido, de la consulta y maternas.

En relación al RN se registró: edad gestacional, peso al nacimiento, sexo, patología perinatal, comorbilidades, edad en días al alta del alojamiento, edad al momento de la consulta, alimentación.

Con respecto a la consulta: fecha, hora, motivo de consulta, número de consultas, derivación médica, estudios complementarios, interconsultas, destino del paciente, tiempo de hospitalización, diagnóstico al egreso.

Los diagnósticos al egreso se agruparon en tres categorías: 1) consultas sobre aspectos de puericultura (dificultades en la lactancia, caída del cordón, llanto, dudas del cuidado del niño); 2) procesos fisiológicos (pseudomenstruación, uratos en la orina, regurgitaciones, eritema toxoalérgico, ictericia, acné neonatal); 3) patología 


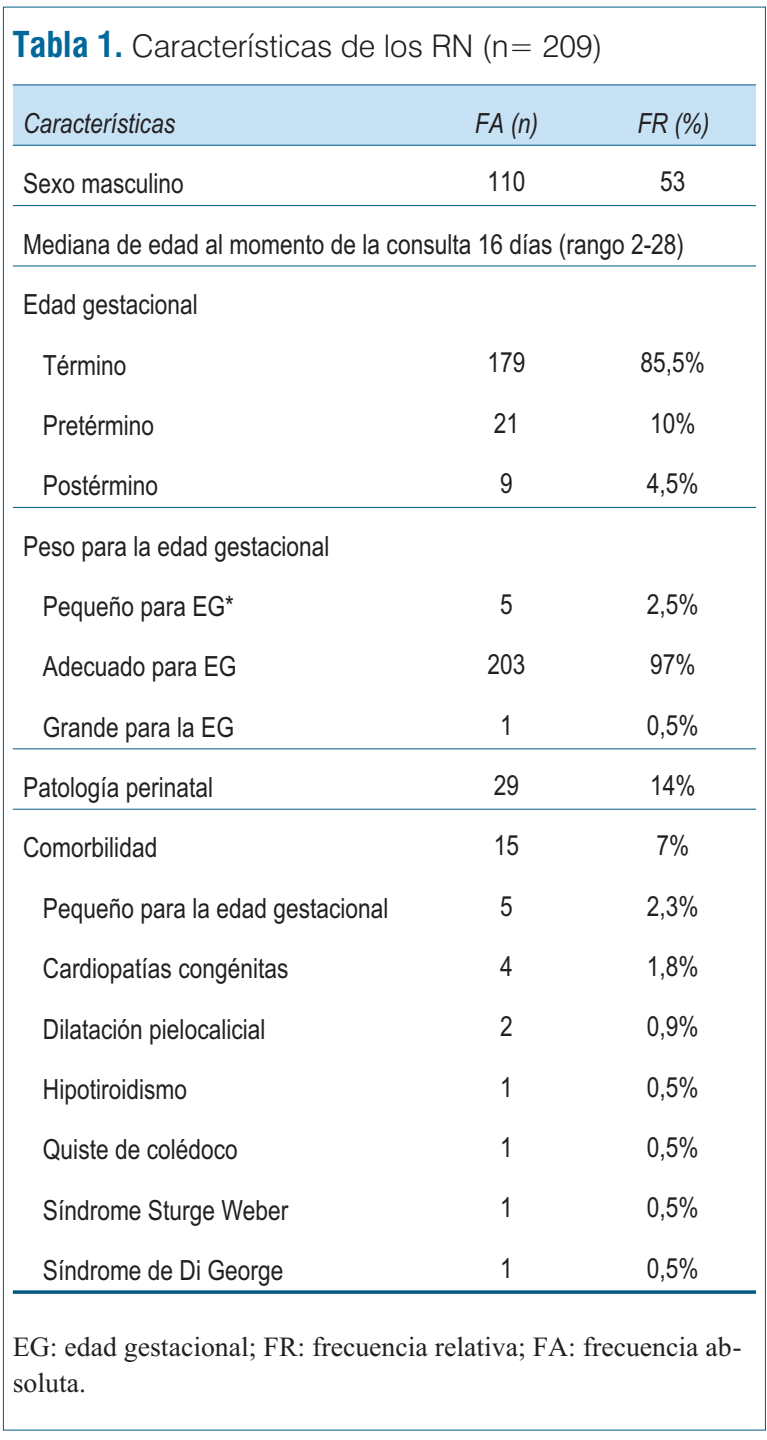

aguda. En relación a la madre: edad, número de hijos, nivel de instrucción, educación sobre puericultura durante el embarazo o puerperio, asistencia a encuentros educativos prenatales, temas que recordaba, entrega de información por escrito sobre puericultura al egreso y opinión personal sobre la utilidad de las instancias formativas. Estos datos fueron obtenidos mediante entrevista telefónica a las madres. Durante el embarazo se realizan actividades institucionales de preparación para el parto y puerperio, además en el alojamiento madre-hijo suele entregarse información por escrito sobre aspectos de puericultura. Por ello se incluyeron preguntas acerca de estos aspectos: asistencia a las actividades, temas tratados en dichas instancias que recordara, así como su opinión acerca de la utilidad de las mismas (respuesta dicotómica sí o no en esta última).

La captación de las consultas se hizo a través del Departamento de Estadística de la institución. Los datos fueron recabados mediante revisión de las historias clínicas electrónicas complementada con entrevista telefónica a las madres.

Las variables continuas se describieron mediante media, mediana y rango y las discretas con frecuencias relativas y absolutas. En la comparación de variables se utilizó X2. Se consideró significativa $\mathrm{p}<0,05$. Para el análisis estadístico se utilizó Epi InfoTM 3.5.4.

El protocolo fue aprobado por el Comité de Ética de la Institución. Se resguardó la confidencialidad de los datos en todas las etapas del estudio.

\section{Resultados}

Se registraron 237 consultas correspondientes a 209 $\mathrm{RN}, 87 \%(\mathrm{n}=182)$ consultaron una vez, $12,5 \%(\mathrm{n}=26)$ dos veces y $0,5 \%$ (un paciente) tres veces.

En este período las consultas neonatales representaron el $1 \%$ de las consultas anuales $(n=24.882)$.

El 53\% ( $\mathrm{n}=110)$ de los $\mathrm{RN}$ eran de sexo masculino, mediana de edad al momento de la consulta 16 días (rango 2-28 días), mediana de edad gestacional al nacer 38 semanas (rango 32-41 semanas). El 85,5\% $(\mathrm{n}=179)$ eran términos y el $97 \%(\mathrm{n}=203)$ adecuados para la edad gestacional.

El 14\% $(n=29)$ presentó patología perinatal: $17 / 29$ ictericia, 7/29 distrés respiratorio, $3 / 29$ depresión neonatal, 3/29 infección/sepsis, 2/29 hipotermia, 1/29 hipoglicemia, 1/29 colestasis, $1 / 29$ conflicto de grupo sanguíneo mayor y $1 / 29$ mal ascenso ponderal. Presentaban comorbilidad el 7\% ( $\mathrm{n}=15), 4 / 15$ cardiopatías congénitas. Las características de los RN se muestran en la tabla 1.

El mes con mayor número de consultas fue setiembre $(12 \%, \mathrm{n}=29)$ y el mes con menos consultas mayo $(5 \%, \mathrm{n}=12)$. No se observó predominio estacional. El $59 \%(n=140)$ de las consultas ocurrieron entre las 8 y las 18 horas.

La mediana de estadía en el SU fue 1,5 horas, media 2 horas (rango 15 minutos-19 horas). El 69\% (n=144) de los RN consultaron en forma espontánea y el 31\% $(n=65)$ derivados por médico.

Los motivos de consulta más frecuentes fueron: síntomas respiratorios $21 \%(\mathrm{n}=51)$, digestivos $14,8 \%$ $(\mathrm{n}=35)$, oftalmológicos $10,5 \% \quad(\mathrm{n}=25)$, llanto $7 \%$ $(n=16)$, fiebre $6,5 \%(n=15)$. Los motivos de consulta y sus frecuencias se muestran en la tabla 2.

Considerando los diagnósticos al egreso del SU se clasificaron en los 3 grupos mencionados: $29 \%(n=69)$ grupo $1,22 \%(\mathrm{n}=51)$ grupo 2 y $49 \%(\mathrm{n}=117)$ grupo 3 . Los diagnósticos al egreso del $\mathrm{SU}$ de los RN con patología aguda (grupo 3) se muestran en la tabla 3.

Con respecto al destino al egreso de las consultas, en $72,5 \%(n=172)$ se otorgó alta domiciliaria y en $27,5 \%$ $(\mathrm{n}=65)$ se indicó hospitalización: 51 en Unidad Neona- 
Tabla 2. Motivos de consulta de los RN $(n=237)$

\begin{tabular}{lcc}
\hline Motivo de consulta & $F A(n)$ & $F R(\%)$ \\
\hline Síntomas respiratorios & 51 & $21 \%$ \\
Síntomas digestivos & 35 & $14,8 \%$ \\
Síntomas oftalmológicos & 25 & $10,5 \%$ \\
Llanto & 16 & $7 \%$ \\
Fiebre & 15 & $6,5 \%$ \\
Lesiones de piel & 15 & $6,5 \%$ \\
Alteraciones en el cordón umbilical & 13 & $5,5 \%$ \\
Ictericia & 11 & $4,6 \%$ \\
Sofocación/apnea/cambios de coloración & 11 & $4,6 \%$ \\
Dificultades en la alimentación & 9 & $3,8 \%$ \\
Tumoraciones & 9 & $3,8 \%$ \\
Mal ascenso ponderal & 9 & $3,8 \%$ \\
Repetición de preparados para lactante & 5 & $2 \%$ \\
Traumatismo encefalocraneano & 3 & $1,3 \%$ \\
Alteraciones urinarias & 3 & $1,3 \%$ \\
Otros & 7 & $3 \%$ \\
\hline
\end{tabular}

FR: frecuencia relativa; FA: frecuencia absoluta.

tal, 12 en cuidados moderados y uno en Unidad de Cuidado Intensivo; dos RN del grupo 1 por escaso incremento ponderal, seis del grupo 2 por ictericia sin conflicto que requirió fototerapia y $56 \mathrm{RN}$ del grupo 3 .

Se realizó encuesta telefónica al 89,5\% $(n=187)$ de las madres. La mediana de edad fue 31 años (rango 16 a 44 años). El 26\% ( $\mathrm{n}=48)$ cursó enseñanza terciaria, $45,5 \%(\mathrm{n}=85)$ secundaria, $28 \%(\mathrm{n}=53)$ Universidad del Trabajo (UTU), y $0,5 \%(\mathrm{n}=1)$ primaria. Concurrieron a clases de cuidados del recién nacido como parte de las actividades preparatorias para parto y puerperio el $73 \%$ $(\mathrm{n}=137)$, de ellas 95,7\% $(\mathrm{n}=132)$ la consideraron de utilidad.

Los contenidos que recordaban como más significativos fueron: lactancia 62/137, higiene del RN 24/137, cuidados generales del RN 18/137, sueño seguro 11/137, salud bucal 10/137 y cuidados del cordón umbilical 9/137. Nueve madres no recordaban ninguno de los temas tratados. El 60\% $(\mathrm{n}=112)$ refirió haber recibido información escrita en el alojamiento madre-hijo previo al alta.

Los diagnósticos al egreso de los pacientes que fueron hospitalizados del grupo 3 se muestran en la tabla 4.
Tabla 3. Diagnósticos al egreso del SU de los RN del grupo 3 ("patología aguda") ( $n=117)$.

\begin{tabular}{lcc}
\hline Diagnósticos & $F A(n)$ & $F R(\%)$ \\
\hline Infección respiratoria aguda alta & 29 & $25 \%$ \\
Conjuntivitis & 21 & $18 \%$ \\
Bronquiolitis & 13 & $11 \%$ \\
Apnea/ALTE/BRUE & 11 & $9,5 \%$ \\
Fiebre sin foco clínico evidente & 10 & $8,5 \%$ \\
Infección de piel & 5 & $4,2 \%$ \\
Traumatismos & 4 & $3,4 \%$ \\
Vómitos & 4 & $3,4 \%$ \\
Varios & 20 & $17 \%$ \\
\hline $\begin{array}{l}\text { FR: frecuencia relativa; FA: frecuencia absoluta; ALTE: evento } \\
\text { de aparente amenaza a la vida; BRUE: episodio breve resuelto } \\
\text { inexplicado. }\end{array}$
\end{tabular}

Tabla 4. Diagnósticos al egreso de los RN del grupo 3 hospitalizados ("patología aguda") $(n=56)$.

\begin{tabular}{lcc}
\hline Diagnósticos & $F A(n)$ & $F R(\%)$ \\
\hline Bronquiolitis & 16 & 28,6 \\
Infección respiratoria aguda alta & 9 & 16 \\
Fiebre sin foco clínico evidente & 8 & 14,2 \\
Apnea/ALTE/BRUE & 7 & 13 \\
RGE & 5 & 9 \\
Traumatismos(TEC, politraumatizado) & 3 & 5 \\
Infección piel & 2 & 4 \\
Infección urinaria & 1 & 1,7 \\
Tos coqueluchoide & 1 & 1,7 \\
Mastitis & 1 & 1,7 \\
Estenosis hipertrófica del píloro & 1 & 1,7 \\
Insuficiencia cardíaca & 1 & 1,7 \\
Alergia proteínas leche vaca & 1 & 1,7 \\
\hline & & \\
ALTE: evento de aparente amenaza a la vida; BRUE: episodio \\
breve resuelto inexplicado; RGE: reflujo gastroesofágico; FR: fre- \\
cuencia relativa; FA: frecuencia absoluta.
\end{tabular}

Se realizaron interconsultas con especialistas en $10 \%$ de las consultas $(\mathrm{n}=23)$ y estudios de laboratorio $\mathrm{y} / \mathrm{o}$ imágenes en $33 \%(\mathrm{n}=80)$.

En la tabla 5 se compara la distribución de algunas variables entre los RN sin y con patología aguda. La fre- 
Tabla 5. Comparación del grupo de RN sin y con patología.

\begin{tabular}{lccc}
\hline Variable & RN Grupos 1 y 2 & RN Grupo 3 & $p$ \\
\hline Prematurez & $7 / 120$ & $13 / 117$ & NS \\
Patología perinatal & $17 / 120$ & $22 / 117$ & NS \\
Madre primigesta & $57 / 120$ & $46 / 117$ & NS \\
Permanencia en SU <2 horas & $78 / 120$ & $64 / 117$ & NS \\
Derivación por médico & $43 / 120$ & $30 / 117$ & NS \\
Estudios complementarios & $24 / 120$ & $56 / 117$ & $<0,05$ \\
Interconsultas & $6 / 120$ & $17 / 117$ & $<0,05$ \\
Hospitalización & $8 / 120$ & $56 / 117$ & $<0,05$
\end{tabular}

FR: frecuencia relativa; FA: frecuencia absoluta.

cuencia de interconsultas, de estudios de laboratorio y/o imágenes y la tasa de hospitalización fue significativamente mayor en el grupo de RN con patología (grupo 3).

La media de días de hospitalización fue 3 días y 12 horas, mediana 3, moda de 2 días (rango 1-14).

No se registraron fallecimientos en el período de estudio

\section{Discusión}

Esta representa la primera comunicación que permite cuantificar y caracterizar los RN que consultan en el SU de este prestador integral de salud privado. Por otro lado, dadas las escasas comunicaciones locales al respecto, aporta insumos para abordar dificultades o problemas que pueden ser comunes en otros prestadores del sistema.

Se destaca que la prevalencia de las consultas neonatales en el SU es baja, similar a la comunicada en estudios previos locales e inferior al de algunas series internacionales Si bien, el número de consultas no representa un problema, la importancia radica fundamentalmente en la elevada vulnerabilidad de este grupo etario que exige evaluación minuciosa y abordaje oportuno y adecuado $^{(1-3,8,11,14)}$.

La media de edad al momento de la consulta, el ligero predominio del sexo masculino y el porcentaje de prematurez son similares a los observados en otras se$\operatorname{ries}^{(1,3,7,11)}$.

Las consultas fueron más frecuentes en horarios diurnos en los que están disponibles los consultorios de atención primaria. Este hallazgo difiere de otros estudios en los que las consultas de $\mathrm{RN}$ predominan fuera de los horarios de consulta ambulatoria habitual ${ }^{(3)}$.
Respecto a los motivos de consulta y diagnóstico, varias publicaciones postulan que en la mayoría de los casos corresponden a patologías banalrs e inespecíficas que no requieren la realización de estudios complementarios ni hospitalización ${ }^{(1-11)}$. En esta serie, aproximadamente la mitad de los pacientes no tenían patología aguda o consultaban por cambios fisiológicos de este período de vida. Este hallazgo permite inferir que una proporción importante de las consultas en el SU son injustificadas y potencialmente evitables.

$\mathrm{Al}$ considerar las patologías agudas más frecuentes, se destacan las infecciones respiratorias agudas altas, las conjuntivitis y bronquiolitis. Las infecciones respiratorias constituyen un motivo frecuente de consulta y hospitalización en toda la población pediátrica. La conjuntivitis neonatal representa una causa frecuente de consulta con incidencias reportadas de hasta $12 \%$ en algunas series. En un estudio local, realizado por Notejane y colaboradores, se reportó una tasa de hospitalización por conjuntivitis neonatal de aproximadamente $5 \%{ }^{(15,16)}$. En esta serie no hubo hospitalizaciones por esta causa.

Al analizar la tasa de hospitalización de los RN, se destaca que triplica la tasa global de ingresos de menores de 15 años en ese SU. El estudio de Fernández Ruiz analiza una serie de neonatos de características similares encontrando una tasa hospitalización muy superior a la tasa de la población pediátrica general ${ }^{(1,3,7,11)}$.

Al considerar los $\mathrm{RN}$ que consultaron por patologías agudas, aproximadamente la mitad fueron hospitalizados. El motivo más frecuente de hospitalización fueron las infecciones respiratorias. Se debe considerar que los $\mathrm{RN}$ dadas sus características anatomofisiológicas e inmunológicas, pueden desarrollar formas clínicas más severas y presentar apneas o cambios de coloración, en- 
tre otros. En la bronquiolitis, los neonatos son considerados un grupo de riesgo de mala evolución con mayor incidencia de insuficiencia respiratoria y de requerimientos de medidas de soporte respiratorio ${ }^{(15)}$.

En esta serie, la referencia por médico al SU no se asoció a patología aguda o ingreso hospitalario ${ }^{(1,11)}$. El principal motivo de referencia fue solicitud de estudios complementarios de laboratorio e imágenes.

La mayoría de las consultas corresponden a $\mathrm{RN}$ sanos, sin antecedentes. La baja frecuencia de prematurez y comorbilidad podría estar vinculada con la existencia de un programa institucional de seguimiento ambulatorio diferenciado para los $\mathrm{RN}$ con estos antecedentes.

Llama la atención que no hubo diferencia significativa entre madres primerizas y multigestas en relación a las consultas en el SU y a las hospitalizaciones. La multiparidad se ha asociado en otras series a una mayor tendencia a presentar consultas justificadas en el SU, lo que no se evidenció en este estudio ${ }^{(8)}$.

No se observaron diferencias significativas en la estadía en el SU entre los diferentes grupos de RN. Es posible que la anamnesis detallada, la realización de un examen físico minucioso y la necesidad de realizar observación en algunos casos, explique que aun ante consultas de poca complejidad, el tiempo de asistencia de los RN sea considerable. Además, el rápido acceso a la hospitalización evita períodos de espera prolongados en el SU. Esto es adecuado a la prioridad y nivel de triage que se designa a este grupo etario. Los tiempos de espera recomendados entre el registro administrativo y la clasificación es de 10 minutos. Para la prioridad III de asistencia (mínima designada a RN) el tiempo de espera seguro hasta la atención médica es de 30 minutos ${ }^{(7,13)}$.

Finalmente, a pesar que la mayoría de las madres asistieron a las capacitaciones durante el embarazo sobre cuidados del RN, las consideraron de utilidad, y recibieron información escrita en el alojamiento madre-hijo, una importante proporción de las consultas fueron motivadas por aspectos de puericultura o procesos fisiológicos que debieron ser abordados en dichas instancias. Como estrategias de mejora institucional, será necesario revisar los contenidos temáticos de las instancias de capacitación, la metodología utilizada en dichos talleres, reforzar las estrategias de comunicación así como los contenidos de los primeros controles de los $\mathrm{RN}$ en el primer nivel de atención.

Este estudio presenta como debilidades el tipo de diseño utilizado, ya que la recolección retrospectiva de los datos, dificulta la obtención de los mismos para algunas variables e introduce sesgos como el de memoria. Por otro lado, su realización en un prestador privado de salud impide la generalización de los resultados a la población local de neonatos. A pesar de estas debilidades, re- presenta la primera comunicación nacional que aborda específicamente las consultas neonatales en el SU, por lo que constituye un importante insumo y el punto de partida para futuras investigaciones con otro tipo de diseño y que incluyan usuarios de los subsectores público y privado de la salud.

\section{Conclusiones}

En esta serie predominaron las consultas de RN sin patología aguda, potencialmente evitables. A pesar de ello, debido a la elevada vulnerabilidad de este grupo etario, el pediatra de urgencias debe realizar una evaluación minuciosa en estos niños. Es probable que, facilitando el acceso al primer nivel de atención y completando la información que se brinda a los padres previo al parto y durante su estadía en maternidad, pueda disminuir la frecuencia de estas consultas. Conocer los problemas de salud de los RN y las principales preocupaciones de los padres constituye un importante insumo para implementar estrategias de mejora y optimizar la utilización de los recursos.

\section{Abstract}

Introduction: the number of newborn presentations to the Pediatric Emergency Department is gradually increasing. However, not all cases are caused by significant risks.

Objective: to describe the characteristics of newborn presentations to the pedriatric emergency department of a comprehensive health care provider between January 1 and December 31, 2018.

Method: descriptive study by analysing medical records and telephone interviews to mothers. Newborn, maternal and consultation variables were analysed.

Results: 237 presentations were recorded, 209 of which corresponded to newborns, male neonates 53\%, average age was 16 days. Diagnostics upon discharge were classified into three categories: 1) consultations associated to childcare $(29 \%)$, 2) physiological processes (22\%) and severe conditions (49\%). Hospitalization rate was $27 \%$. 64 newborns were hospitalized: 2 of them belonging to group 1 (low weight gain); 6 to group 2 (jaundice); 56 to group 3. Average hospital stay was 3 days (range 1-14). No deaths were recorded.

Conclusions: most consultations were caused by non-severe conditions which were potentially avoidable. However, given the high vulnerability of this group of patients, emergency pediatricians must carefully assess these infants. It may be possible that the frequency of these consultations drops by making access to the first level of care easier, and by providing parents with more complete information. Learning about the health problems that are typical of newborns and the main con- 
cerns of parents constitutes an important input for the implementation of improvement strategies and to optimize the use of resources.

\section{Resumo}

Introdução: as consultas de recém-nascido (RN) são cada vez mais frequentes nos serviços de emergência (PS). No entanto, nem sempre correspondem a problemas com risco significativo.

Objetivo: descrever as características das consultas de RNs no PS de uma instituição privada de saúde integral no período 1 de janeiro a 31 de dezembro de 2018.

Metodologia: estudo descritivo, por meio de revisão de prontuários e entrevista telefônica com as mães. Analisaram-se RN, consulta e variáveis maternas.

Resultados: foram registradas 237 consultas, correspondendo a 209 RNs, 53\% do sexo masculino, média de idade de 16 dias. Os diagnósticos de alta foram agrupados em três categorias: 1) consultas relacionadas aos aspectos da puericultura $29 \%$, 2) processos fisiológicos $22 \%$ e 3 ) patologias agudas $49 \%$. A taxa de internação foi de $27 \%$. Foram admitidos 64 RNs: 2 do grupo 1 (pequeno aumento de peso); 6 do grupo 2 (icterícia); 56 do grupo 3. O tempo médio de internação foi de 3 dias (variação 1-14). Não houve mortes.

Conclusões: predominaram as consultas de recém-nascidos sem patologia aguda, potencialmente evitáveis. Apesar disso, devido à alta vulnerabilidade dessa faixa etária, o pediatra do pronto-socorro deve realizar uma avaliação criteriosa dessas crianças. É provável que, ao facilitar o acesso ao primeiro nível de atendimento e ao preencher as informações prestadas aos pais, seja possível diminuir a frequência dessas consultas. Conhecer os problemas de saúde dos recém-nascidos e as principais preocupações dos pais constitui um importante insumo para implementar estratégias de melhoria e otimizar o uso de recursos.

\section{Bibliografía}

1. Fornes Vivas R, Mustiens Bellot R, Navarro Juanes A, Robledo Díaz L, Carvajal Roca E. ¿Es adecuada la utilización del servicio de urgencias en el período neonatal? Rev Pediatr Aten Primaria 2017; 19(76):301-9.

2. Zimmer K, Walker A, Minkovitz C. Epidemiology of pediatric emergency department use at an urban medical center. Pediatr Emerg Care 2005; 21(2):84-9.

3. Fernández Ruiz C, Trenchs Sainz de la Naza V, Curcoy Barcenilla A, Lasuen del Olmo N, Luaces Cubells C. Asistencia a neonatos en el servicio de urgencia de un hospital terciario. An Pediatr (Barc) 2006; 65(2):123-8.

4. O'Donnell H, Colman G, Trachtman R, Velazco N, Racine A. Impact of newborn follow-up visit timing on subsequent ED visits and hospital readmissions: an instrumental variable analysis. Acad Pediatr 2014; 14(1):84-91.

5. Kennedy T, Purcell L, LeBlanc J, Jangaard K. Emergency department use by infants less than 14 days of age. Pediatr Emerg Care 2004; 20(7):437-42.

6. Millar K, Gloor J, Wellington N, Joubert G. Early neonatal presentations to the pediatric emergency department. Pediatr Emerg Care 2000; 16(3):145-50.

7. Flanagan C, Stewart M. Factors associated with early neonatal attendance to a paediatric emergency department. Arch Dis Child 2014; 99(3):239-43.

8. Droz N, Chaleard J, Laurent M, Mandelbrot L, Cojocaru B. Les consultations des nouveau-nés aux urgences pédiatriques. Soins Pediatr Pueric 2018; 39(303):32-7.

9. Richier P, Gocko X, Mory O, Trombert-Paviot B, Patural H. Étude épidémiologique des consultations précoces de nouveau-nés aux services d'accueil des urgences pédiatriques. Arch Pediatr 2015; 22(2):135-40.

10. Cabrera S, Iglesias J, Bernárdez I, Marín M, Hidalgo M, Braverman A. Principales causas de visita de neonatos al servicio de urgencias de un hospital privado. An Med (Mex) 2016; 61(4):251-5. Disponible en: https://www.medigraphic.com/pdfs/abc/bc-2016/bc164c.pdf. [Consulta: 13 noviembre 2020].

11. Batu E, Yeni S, Teksam O. The factors affecting neonatal presentations to the pediatric emergency department. J Emerg Med 2015; 48(5):542-7.

12. Pérez Solís D, Pardo R, Fernández A, Ibáñez A, Prieto S, Fanjul J. Atención a neonatos en una unidad de urgencias pediátricas. An Pediatr (Barc) 2003; 59(1):54-8.

13. Mezquita M, Pavlicich V, Luaces C. El sistema español de triage en la evaluación de los neonatos en las urgencias pediátricas. Rev Chil Pediatr 2017; 88(1):107-12.

14. Assandri E, Ferreira M, Bello O, de Leonardis D. Hospitalización neonatal desde el servicio de urgencias en un centro hospitalario de Uruguay. An Pediatr (Barc) 2005; 63(5):413-7. 
15. Boyadjian S, Notejane M, Assandri E, Pujadas M, Pírez C. Bronquiolitis en neonatos. Experiencia de cuatro años en un hospital de referencia nacional. Arch Pediatr Urug 2015; 86(4):265-72.
16. Notejane M, Casuriaga A, Giachetto G. Hospitalizaciones por conjuntivitis neonatal infecciosa en un centro de referencia de Uruguay: características clínicas y evolutivas. Rev Hosp Jua Mex 2019; 86(1):26-32.

\section{Contribución de autores}

Mariana Más, ORCID 0000-0001-8531-6810. Concepción, diseño, recolección de datos, procesamiento de resultados, discusión, escritura.

Ana Casuriaga, ORCID 0000-0002-1122-5147. Concepción, diseño, recolección de datos, procesamiento de resultados, discusión, escritura.

Carina Giacoia, ORCID 0000-0002-7408-8768. Recolección de datos, procesamiento de resultados, discusión y escritura. Lucía Centena, ORCID 0000-0001-9403-8541. Recolección de datos, procesamiento de resultados, discusión y escritura. Magdalena López, ORCID 0000-0001-5995-4149. Recolección de datos, procesamiento de resultados, discusión y escritura. Yelenna Ramírez, ORCID 0000-0002-6213-3525. Procesamiento de resultados, discusión y escritura.

Gustavo Giachetto, ORCID 0000-0003-3775-4773. Concepción, diseño, discusión, escritura y corrección. 